\title{
Ultrafast spectroscopy of single molecules
}

\author{
Erik van Dijk ${ }^{2}$, Mark te Paske ${ }^{2}$, Jordi Hernando ${ }^{3}$, \\ Jacob Hoogenboom ${ }^{2}$, Maria Garcia-Parajo ${ }^{4,5}$, and Niek van Hulst ${ }^{1,5, *}$ \\ ${ }^{1}$ ICFO - Institut de Ciències Fotòniques, Av. Canal Olímpic, 08860 Castelldefels, Spain \\ E-mail: Niek.vanHulst@ICFO.es www.ICFO.es \\ ${ }^{2}$ Applied Optics group, MESA+ Institute for Nanotechnology, University of Twente, \\ 7500AE Enschede, the Netherlands \\ ${ }^{3}$ Dept. de Química, Universitat Autónoma Barcelona, 08193 Cerdanyola del Valles, Spain \\ ${ }^{4}$ Lab. of NanoBioEngineering, Barcelona Scientific Park (PCB), 08028 Barcelona, Spain \\ ${ }^{5}$ ICREA - Institució Catalana de Recerca i Estudis Avançats, 08015 Barcelona, Spain
}

\begin{abstract}
We present a single-molecule study on femtosecond dynamics in multichromophoric systems, combining fs pump-probe, emission-spectra and fluorescencelifetime analysis. At the single molecule level a wide range of exciton delocalisation lengths and energy redistribution times is revealed. Next, two color pump-probe experiments are presented as a step to addressing ultrafast energy transfer in individual complexes.
\end{abstract}

\section{Introduction}

Until recently pico- and femto-second experiments have been restricted to large populations of molecules, yielding only average molecular response. In 2004 we bridged the gap between "ultrafast" and "single molecule" detection by a novel single-molecule pump-probe (SM2P) method [1]. Two femtosecond pulses excite a chosen individual molecule and when the two pulses are separated more than the typical molecular decay time, the single molecular emission is stronger than when the pulses are coincident in time. The ultrafast response time varies strongly from molecule to molecule, indicating that the intramolecular dynamics is largely affected by conformational variation and heterogeneity of the immediate environment [2].

Ultrafast detection of single entities is particularly relevant for fs processes in complex systems, such as light harvesting complexes, autofluorescent proteins, photonic polymers, etc. As a first step in this direction we have focused on coupled molecules, where we find longer decay times and discrete jumps in femtosecond response, reflecting sudden change in coupling of the quantum coupled system. Here we focus on the trimer complex of the tetraphenoxyperylene diimide dye (TPD, Fig. 1) [3].

Secondly, to address ultrafast processes between specific states (i.e. fluorescence energy transfer and charge transfer) it is of course crucial to extent our degenerate SM2P method towards a two color scheme. Here we present a two color pump-dump approach, in which the spontaneous emission is reduced due to competitive stimulated emission depletion [4]. So far the dump efficiency of fs pulses is restricted by long-lived vibrational states. 


\section{Pump-probe spectroscopy on single complexes}

Single molecule time traces of TPD complexes reveal the occurrence of multilevel intensities, due to sequential photodamaging of the constituent chromophores in the complex $[3,5,6]$. The jumps in fluorescence intensity correlate with changes in fluorescence lifetime $\left(\tau_{\mathrm{F}}\right), 3.9 \mathrm{~ns}$ for the trimer to $6.5 \mathrm{~ns}$ for the monomer, as the oscillator strength concentrates on the lowest exciton state: superradiance $[5,6]$. Fig. 1 shows the effect of exciton delocalization on the emission spectrum. Besides red-shift, the vibronic component at $\lambda \sim 660 \mathrm{~nm}(1-0$ band) is weaker. The delocalized nature of the exciton is responsible for decreased coupling between the electronic state and the vibrational mode $[7,8]$.
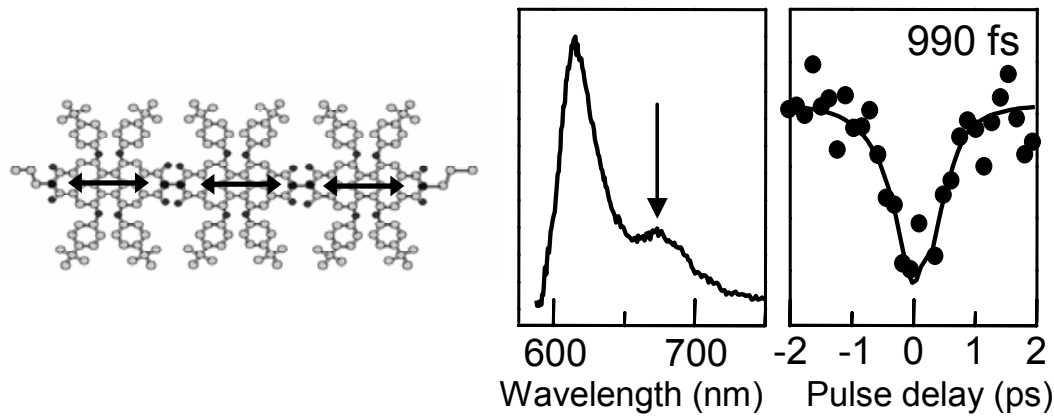

Fig. 1. The trimer complex of tetraphenoxy-perylene diimide (TPD). Emission of spectrum of a single TPD molecule, with the $0-0$ and 1-0 emission bands at $\lambda \sim 610 \mathrm{~nm}$ and $\lambda \sim 660 \mathrm{~nm}$, respectively. SM2P dip for an intact excitonic trimer, where the solid line shows a fit which recovers a redistribution time of 990 fs for this particular molecule, as compared to $\sim 80 \mathrm{fs}$ for the perylene diimide monomer.

Coupling between electronic and vibrational degrees of freedom also governs the ultrafast dynamics of excited states. Fig. 1 shows the result of applying SM2P to a single trimer. A clear dip in fluorescence intensity at zero delay is visible. At $\Delta \tau=0$ the emission is not increased since the transition is already saturated. For $\Delta \tau>0$, coupling to phonons leads to relaxation of the initially excited state and fluorescence is increased $[1,2]$. The width of the dip reflects the ultrafast processes connected with the initially excited state, 990 fs in Fig. 1. Interestingly, the redistribution time increases with the number of interacting molecules, indicating a decrease in phonon coupling efficiency upon delocalization, consistent with the spectral data.

\section{Two color pump-probe spectroscopy}

A two color scheme has been implemented to address specific initial and final states. The pump pulse is followed by a saturating dump pulse that depletes the 
excited state, in direct competition with the generated fluorescence [4]. Indeed fluorescence can reduced (Figure 2); however the efficiency depends on the spectral-temporal overlap of the dump pulse with the involved vibrational states. Only for stretched pulses ( $>4 \mathrm{ps}$ ) an appreciable reduction in fluorescence occurs, while the recovery time tends towards the fluorescence lifetime. The timebandwidth content of the dump pulse requires further fine-tuning.

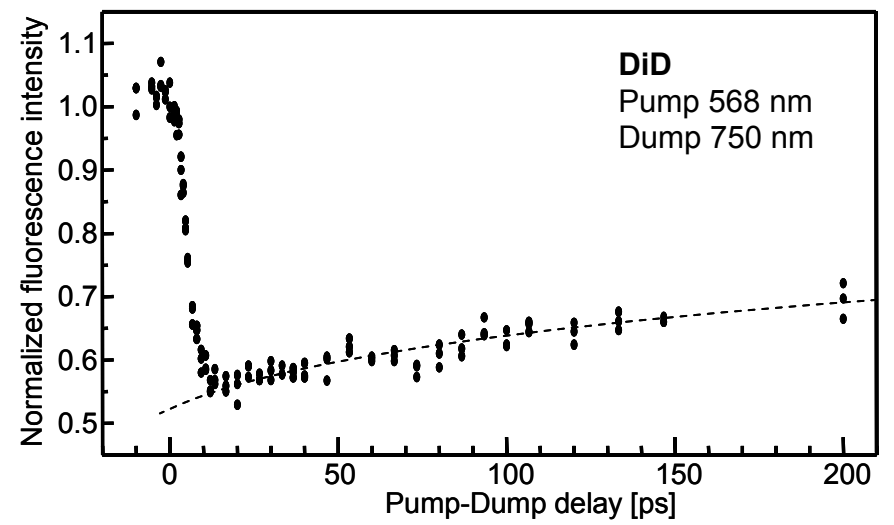

Fig. 2. Two color pump-probe (dump) spectroscopy on DiD (1,1'-dioctadecyl-3,3,3',3'tetra-methyl-indodicarbocyanine) molecules. Pump pulse, $250 \mathrm{fs}$, at $568 \mathrm{~nm}$; dump pulse stretched to $5 \mathrm{ps}$, at $750 \mathrm{~nm}$.

\section{Conclusions}

We have presented femtosecond detection of single excitonic complexes, at room temperature. Excitonic delocalization increases the intramolecular energy redistribution time. The ultrafast single molecule detection has applications in molecular biology, molecular photonics and quantum-optics.

Acknowledgements. Presented work has been financed by the Dutch Foundation for Fundamental Research of Matter (FOM) (E.v.D.), the Spanish MEC Programs Ramon y Cajal (J.H.) \& Plan Nacional MAT2006-08184 and the German VWStiftung (JP.H.).

\section{References}

1 E.M.H.P. vanDijk, J. Hernando, et al., Phys. Rev. Lett. Vol. 94, 078302, 2005.

2 E.M.H.P. van Dijk, J. Hernando et al., J. Chem. Phys. Vol. 123, 064703, 2005.

3 J. Hernando, E.M.H.P. van Dijk, et al. Submitted.

4 S.W. Hell Nature Biotechnology Vol. 21, 1347, 2003.

5 M. Lippitz, C.G. Hubner, et al., Phys. Rev. Lett. Vol. 92, 103001, 2004

6 J. Hernando, J.P. Hoogenboom, et al., Phys. Rev. Lett. Vol. 93, 236404, 2004.

7 F. C. Spano, J. Chem. Phys. Vol. 116, 5877, 2002.

8 Y.C. Cheng and R.J. Silbey, Phys. Rev. Lett. Vol. 96, 028103, 2006. 